Case report

\title{
Negative pressure pulmonary edema in the prone position:
} a case report

\author{
Hesham Omar ${ }^{1}$, Jaya Kolla ${ }^{2}$, Amrat Anand ${ }^{3}$, Willem Nel ${ }^{3}$, \\ Devanand Mangar ${ }^{2}$ and Enrico Camporesi ${ }^{4 *}$
}

\author{
Addresses: ${ }^{1}$ Department of Cardiovascular Medicine, Cairo University Hospital, Cairo, Egypt, Visiting Scholar at Tampa General Hospital, \\ Florida Gulf to Bay Anesthesiology Associates, PA, Tampa, Florida, USA \\ ${ }^{2}$ Research Associate, Tampa General Hospital, Florida Gulf to Bay Anesthesiology Associates, PA, Tampa, Florida, USA \\ ${ }^{3}$ Florida Gulf to Bay Anesthesiology Associates, PA, Tampa, Florida, USA \\ ${ }^{4}$ Department of Surgery/Anesthesiology, University of South Florida, Tampa, FL and Florida Gulf to Bay Anesthesiology Associates, PA, \\ Tampa, Florida, USA \\ Email: HO - hesham_engy2008@yahoo.com; JK - jaykolla@yahoo.com; AA - aanand6070@hotmail.com; WN - wjnel@yahoo.com; \\ DM - dmangar@tgh.org; EC* - ecampore@health.usf.edu \\ * Corresponding author
}

Received: 8 December 2008 Accepted: 19 June 2009 Published: 27 July 2009

Cases Journal 2009, 2:8594 doi: 10.4076/1757-1626-2-8594

This article is available from: http://casesjournal.com/casesjournal/article/view/8594

(C) 2009 Omar et al; licensee Cases Network Ltd.

This is an Open Access article distributed under the terms of the Creative Commons Attribution License (http://creativecommons.org/licenses/by/3.0), which permits unrestricted use, distribution, and reproduction in any medium, provided the original work is properly cited.

\begin{abstract}
Acute airway obstruction can result in life - threatening pulmonary edema. It can develop rapidly, without warning, in otherwise healthy patients. Negative pressure pulmonary edema has been described after acute airway obstruction in situations when a patient is breathing against an obstructed airway such as croup, epiglottitis or laryngospasm. In the following case, we observed a rare occurrence of pulmonary edema in a female following sedation in the prone position.
\end{abstract}

\section{Case presentation}

A 49-year-old Caucasian female, with a long history of heavy cigarette smoking and a previous diagnosis of supraventricular tachycardia, for which cardiac ablation was performed 2 years ago, was admitted to the hospital for an elective sacral neuromodulator lead change to treat urinary incontinence. In the operating room, the patient received monitored anesthesia care (MAC) in the prone position. During the procedure she required increasing sedation (IV propofol infusion) and after thirty minutes the patient showed progressive hypercapnia from the nasal cannula sample line, while receiving supplemental oxygen $(4 \mathrm{~L} / \mathrm{min})$. An oral airway was placed, but end-tidal $\mathrm{CO}_{2}$ ranged from
65 to $75 \mathrm{mmHg}$ and did not improve. It was then decided to place a laryngeal mask and to supply positive pressure ventilation; however no improvement of the hypercapnia ensued and we could not provide adequate ventilation. The patient was then emergently intubated to secure an airway and provide adequate ventilation. This required interruption of surgery, turning the patient supine, ventilating oxygen by mask and a succinylcholine bolus. During the intubation process, marked edema above the vocal cords was noticed by an experienced anesthesiologist, allowing only for a smaller sized endotracheal tube to be inserted ( $6 \mathrm{~mm}$ with difficulty, but not a $7 \mathrm{~mm}$ tube). The patient was given dexamethasone $8 \mathrm{mg}$ and diphenhydramine $25 \mathrm{mg}$ for the airway edema. 
Following intubation and after securing the tube in place, we started positive pressure ventilation and returned the patient to the prone position, and the end tidal $\mathrm{CO}_{2}$ returned within normal range. However, within a few minutes, the patient developed bibasilar rales, with abundant frothy, blood tinged secretions appearing in the tube. We maintained the patient prone while the surgical procedure was concluded. The edema fluid was adequately suctioned and abated after 10 minutes of positive pressure ventilation. Postoperatively the patient remained intubated and sedated on a propofol infusion. While in recovery, an arterial blood gas obtained on Synchronized Intermittent Mechanical Ventilation (SIMV) and $\mathrm{FiO}_{2}$ of $30 \%$, showed a $\mathrm{PO}_{2}$ of $92 \mathrm{mmHg}$, $\mathrm{PCO}_{2}$ of $46 \mathrm{mmHg}$, $\mathrm{pH}$ of 7.34 and $\mathrm{HCO}_{3}$ of $25 \mathrm{mEq} / \mathrm{L}$ with $\mathrm{a} \mathrm{SaO}_{2}$ of $97 \%$. Chest X-ray performed after surgery indicated clear lung fields with normal size heart and no evidence of pulmonary edema. The patient's condition steadily improved and she was subsequently extubated two hours later but continued to be closely monitored overnight and was discharged the following day without requiring an ICU stay.

\section{Discussion}

In this case report we describe a case of NPPE following supraglottic obstruction with deepening sedation in the prone position. Supraglottic obstruction was possibly induced by intense inspiratory effort in a patient who was sedated and hypoventilating leading to airway obstruction and subsequently pulmonary edema. Another possibility is malpositioning (or biting) of the LMA causing inspiratory efforts against an obstructed airway, which could be the cause for the observed supraglottic swelling. The apparent absence of gastric contents in the pulmonary edema fluid, the absence of left ventricular dysfunction in a previously performed echocardiogram and the sequence of the described symptoms make the diagnosis of acute NPPE likely.

NPPE is a relatively uncommon intra-operative and postoperative complication and is still a diagnosis by exclusion of other factors. Generally, NPPE occurs when inspiratory effort against an obstruction creates a large intrapulmonary negative pressure which can increase venous return with subsequent increase in the pulmonary capillary hydrostatic pressure. The transudation of fluid from the pulmonary vasculature causes edema formation into the alveolar space $[1,2]$. Normal inspiratory pleural pressure ranges from -5 to $-8 \mathrm{~cm}$ of $\mathrm{H}_{2} \mathrm{O}$, but in instances of obstruction, inspiratory pressures can increase to $-50 \mathrm{~cm}$ of $\mathrm{H}_{2} \mathrm{O}$ and in extreme circumstances have been known to exceed $-100 \mathrm{~cm}$ of $\mathrm{H}_{2} \mathrm{O}$ [3]. Typically, these patients are young athletic males that have well-developed musculature, capable of creating a large negative pressure [4].
NPPE has also been described in detail in children following tonsillectomies. In these patients inspiratory efforts against airway obstruction creates a positive endexpiratory pressure (auto-PEEP) which decreases the transcapillary pressure gradient $\left(\mathrm{P}_{\mathrm{c}}-\mathrm{P}_{\mathrm{i}}\right)$ preventing fluid leak into the interstitium. The sudden relief of the obstruction may lead to loss of the auto-PEEP resulting in a large transcapillary pressure gradient and subsequent pulmonary edema $[2,5,6]$. NPPE has also been described in patients with upper airway obstruction in cases of epiglottitis and croup, foreign body obstruction, airway secretions, following re-expansion of an atelectatic region of the lung or rapid drainage of a pleural effusion, after near strangulation and after oral and pharyngeal surgery. Other presentations have been described after vigorous suction during bronchoscopy, obstructive sleep apnea and biting on the laryngeal mask tube $[2,7,8]$.

NPPE is not only observed during or after anesthesia; numerous reports in the literature describe situations of respiratory loading requiring large inspiratory efforts, as in submerged scuba divers or breath-holding divers after resurfacing and even swimmers $[9,10,11]$. The proposed theory behind such a predisposition is that immersion causes central pooling of blood in the pulmonary circulation, either from the peripheral vasoconstrictive effects of cold water, or via increased venous return as caused by warm water immersion. Ultimately, intense inspiratory efforts while immersed in water may create a large negative intrathoracic pressure. The combination of these factors leads to stress failure of pulmonary capillaries and the breakdown of the alveolar wall, causing high-permeability' pulmonary edema or even frank hemorrhage [12].

Treatment of NPPE is usually supportive and includes maintenance of a patent airway and adequate oxygenation. The uses of dexamethasone and diuretics have not shown to be efficacious in prospective studies and may be deleterious [7]. In severe cases endotracheal intubation and mechanical ventilation with PEEP is usually necessary. The prognosis is usually benign, but $11 \%$ to $40 \%$ of the described cases develop life threatening complications, including mortality [2].

In conclusion, negative pressure pulmonary edema is a possible cause of respiratory distress in the operating room, especially in patients breathing spontaneously while in the prone position. Any compromise of the airway in the prone position is difficult to recover. We therefore suggest that these patients receive adequate protection of their airway, comprising aggressive intubation, general anesthesia and continuous ventilatory support. 


\section{Abbreviations}

NPPE, Negative pressure pulmonary edema; PEEP, Positive end expiratory pressure; $\mathrm{P}_{\mathrm{C}}$, Capillary hydrostatic pressure; $\mathrm{P}_{\mathrm{i}}$, Interstitial pressure; LMA, Laryngeal mask airway.

\section{Consent}

Written informed consent was obtained from the patient for publication of this case report. A copy of the written consent is available for review by the Editor-in-Chief of this journal.

\section{Competing interests}

The authors declare that they have no competing interests.

\section{Authors' contributions}

$\mathrm{HO}$ and JK were responsible for drafting and literature search. EC and DM were responsible for diagnosis and management of the case. AA and $\mathrm{WN}$ have made critical suggestions regarding the manuscript. All authors have read and approved the final manuscript.

\section{References}

I. Ferrari LR, Gotta AW: Anesthesia for Otolaryngologic Surgery. In Clinical Anesthesia. 5th edition. Edited by Barash PG, Cullen BF, Stoelting RK Lippincott: Williams and Wilkins; 2006:1000-100I.

2. Thiagarajan RR, Laussen PC: Negative pressure pulmonary edema in children - pathogenesis and clinical management. Pediatr Anesth 2007, 17:307-310.

3. Cascade PN, Alexander GD, Mackie DS: Negative pressure pulmonary edema after endotracheal intubation. Radiology 1993, | 86:67|-675.

4. Holmes JR, Hensinger RN, Wojtys EW: Post-operative pulmonary edema in young, athletic adults. Am J Sports Med I99I, 19:365-37|.

5. Lang SA, Duncan PG, Shephard DAE, Ha HC: Pulmonary oedema associated with airway obstruction. Can J Anaesth 1990, 37:210-218.

6. Scarbrough FE, Wittenberg JM, Smith BR, Adcock DK: Pulmonary edema following postoperative laryngospasm: case reports and review of the literature. Anesth Prog 1997, 44: I I0-1 I6.

7. Devys JM, Balleau C, Jayr C, Bourgain JL: Biting the laryngeal mask: an unusual case of negative pressure pulmonary edema. Can J Anesthes 2000, 47:176-178.

8. Messerschmitt $P$, Stambough JL: Negative pressure pulmonary edema in the arthroscopic patient. Orthopedics 2005, 28:4|3-4I5.

9. Koehle MS, Lepawsky M, McKenzie DC: Pulmonary Oedema of Immersion. Sports Med 2005, 35:183-190.

10. Slade JB, Hattori T, Ray C, Bove AA, Cianci P: Pulmonary Edema Associated with Scuba Diving: Case Reports and Review. Chest 200I, I 20:1686-1694.

II. Beinart R, Matetzky S, Arad T, Hod H: Cold Water-induced Pulmonary Edema. Am J Med 2007, I 20:e3.

12. Mussi RK, Toro IFC: Negative-pressure pulmonary edema and hemorrhage associated with upper airway obstruction. J Bras Pneumol 2008, 34:420-424.

\section{Do you have a case to share?}

\section{Submit your case report today}

- Rapid peer review

- Fast publication

- PubMed indexing

- Inclusion in Cases Database

\section{Any patient, any case, can teach us something}

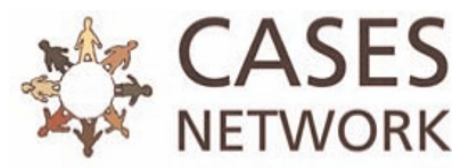

www.casesnetwork.com 